\title{
On the basis set selection for calculations of core-level states: different strategies to balance cost and accuracy
}

Sarangi, Ronit; Vidal, Marta L.; Coriani, Sonia; Krylov, Anna I.

Published in:

Molecular Physics

Link to article, DOI:

$10.1080 / 00268976.2020 .1769872$

Publication date:

2020

Document Version

Peer reviewed version

Link back to DTU Orbit

Citation (APA):

Sarangi, R., Vidal, M. L., Coriani, S., \& Krylov, A. I. (2020). On the basis set selection for calculations of corelevel states: different strategies to balance cost and accuracy. Molecular Physics, 118(19-20), [e1769872]. https://doi.org/10.1080/00268976.2020.1769872

\section{General rights}

Copyright and moral rights for the publications made accessible in the public portal are retained by the authors and/or other copyright owners and it is a condition of accessing publications that users recognise and abide by the legal requirements associated with these rights.

- Users may download and print one copy of any publication from the public portal for the purpose of private study or research.

- You may not further distribute the material or use it for any profit-making activity or commercial gain

- You may freely distribute the URL identifying the publication in the public portal 


\title{
On the basis set selection for calculations of core-level states: Different strategies to balance cost and accuracy
}

\author{
Ronit Sarangi ${ }^{a}$, Marta L. Vidal ${ }^{b}$, Sonia $\mathrm{Coriani}^{b}$, and Anna I. Krylov ${ }^{a}$ \\ ${ }^{a}$ Department of Chemistry, University of Southern California, Los Angeles, CA 90089, USA \\ ${ }^{b}$ DTU Chemistry - Department of Chemistry, Technical \\ University of Denmark, DK-2800, Kongens Lyngby, Denmark
}

We present a study on basis set effects in correlated calculations of core-level states. While it is well recognized that the core-level states require using more extensive basis sets than their valence counterparts, the standard strategy has been to use large contracted basis sets, such as the cc-pVXZ or cc-pCVXZ series. Building upon the ideas of Besley, Gilbert, and Gill [J. Chem. Phys. 130, 124308 (2009)], we show that a much more effective strategy is to use uncontracted bases, such as core or fully uncontracted Pople's basis. The physical grounds behind this approach are explained and illustrated by numerical results. We also discuss other cost-saving strategies, such as virtual space truncation and mixed precision execution.

\section{INTRODUCTION}

Owing to their unique capabilities, spectroscopies exploiting core-level transitions are gaining popularity[1-5]. The transitions involving core electrons are element-specific because of large energy gaps (hundreds of electron-volts) between different edges, yet they are sensitive to their chemical environment. Compact and localized shapes of core orbitals result in local sensitivity, which is particularly important for designing spectroscopic probes of the electronic structure and dynamics in complex environments. As in the case of valence spectroscopies[6], theoretical modeling is crucially important for the interpretation of the experimental spectra. Thus, the progress in experimental techniques, ranging from advanced light sources to table-top X-ray instruments, has stimulated vigorous theoretical developments [7, 8].

At first glance, electronic structure behind valence (UV-VIS) and core-level (X-ray) spectroscopies appear to be similar. For example, modeling photoelectron spectra entails calcula- 
tions of $N$-1-electron states of a neutral molecule. Since the molecular Schrödinger equation contains the solutions for all states, regardless of their energy, one may expect that the same quantum-chemistry method could be used to describe both valence-ionized and core-ionized states. This is, however, not the case: although the Schrödinger equation is the same, the approximations to it, which are used to construct a practical quantum chemistry method (i.e., theoretical model chemistry, in John Pople's terms[9]) may lead to manifestly different outcomes in the valence and core domains.

Theoretical model chemistry[9] is defined by the pair of approximations: one to the manybody problem (correlation treatment) and one to a one-electron basis set used to represent molecular orbitals and construct Slater determinants.

Equation-of-motion coupled-cluster (EOM-CC) theory[10-15] provides an effective and robust treatment of electron correlation and is capable of a treating multiple electronic states on the same footing. Its accuracy can be systematically improved up to the exact limit. These properties make EOM-CC the method of choice for spectroscopy modeling. Challenges in correlated treatment of core-level states and possible solutions have been analyzed in recent reviews [7, 8] and original research papers[16-31]. Particularly effective approach is the extension of the EOM-CC methods to core-level states via the core-valence separation (CVS) scheme[24, 27, 32, 33]. The benchmarks illustrated that CVS-EOM-IP/EE-CCSD [24, 26$28,33,34]$ provides an effective and reliable description of core-ionized and core-excited states, including treatment of non-linear optical properties such as RIXS cross sections[2931 .

The special requirements to one-electron basis sets in calculations of core-level states have been recognized and documented in many papers[16, 20, 35-39]. In a nutshell, obtaining converged and accurate results for core-level states requires considerably larger bases than needed for their valence counterparts. This high sensitivity of the results to the one-electron basis is observed even in uncorrelated calculations, e.g., at the Hartree-Fock level[16]. Its physical origin is a strong perturbation caused by the creation of a core hole as a result of removing or exciting core electrons. To describe the ensuing changes in electronic structure, traditionally referred to as orbital relaxation, sufficiently flexible basis sets are needed.

In the previous CVS-EOM-CC benchmark studies, series of standard contracted basis sets have been tested[35, 40]. In Ref. [35], Coriani and coworkers investigated the convergence with respect to the basis-set size with coupled-cluster methods of increasing complexity 
(CC2, CCSD, CC3, and CCSDT). The largest bases tested in this study were aug-cc-pCV5Z and d-aug-cc-pCV5Z. The authors observed monotonous decrease of the computed ionization and excitation energies towards the experimental values upon increasing the basis-set cardinal number, which illustrates that the target core-level states are more sensitive to the basis set than the ground-state reference. Coriani and co-workers[35] exploited this smooth convergence of the results to extrapolate the computed excitation energies to the complete basis set (CBS) limit. They noted that for ionization energies the aug-cc-pCV5Z basis (the largest used for IEs in their study) appears to be close to the convergence limit, judging from the small differences between this and smaller bases. While additional diffuse functions are required to properly describe core-excited states, they were found to be less important for core-ionized states. The relativistic effects were found to be less sensitive to the basis set[16, 40]. These observations confirm that the main reason for extended basis sets in core-level calculations is due to orbital relaxation.

Here we systematically explore an alternative strategy, used by Gill and coworkers[16] and by us in recent applications[29]. Instead of following the hierarchy of Dunning's bases, optimized to describe electron correlation in ground-state molecules, we build series of basis sets by uncontracting the core and valence functions. We consider Pople's and Dunning's set and show that using uncontracted Pople's bases is much more effective that using Dunning's bases. Our results provide a simple guideline for choosing basis sets for calculations of corelevel states. In addition to effective basis-set choices, we also briefly explore other cost-saving strategies.

\section{STUDY DESIGN, THEORETICAL METHODS, AND COMPUTATIONAL PROTOCOLS}

In this study, we focus on the calculations of ionized states using the fc-CVS-EOM-IPCCSD method[27, 41, 42], with the goal of investigating the ability of various basis sets to describe orbital relaxation effects at a correlated level of theory. By focusing on coreionized states, we can investigate perturbation of the electronic structure due to creation of the core hole. Because excitation of core electrons also creates a core hole, the results should be largely transferable to core-excited states, with the caveat that the calculations of the XAS transitions require additional diffuse functions to describe states of Rydberg 
character. While we provide experimental results when available, our main emphasis is not on the differences relative to the experiment, but rather on the convergence of the theoretical values to the basis-set limit.

To explain the rational behind the design of our study, let us briefly discuss the effects caused by the removal of a core electron. Because core orbitals are compact, they screen the nuclear charge much more effectively than the valence orbitals do. Thus, removing a core electron from an atom is roughly equivalent to increasing the nuclear charge by one, in terms of the Coulomb field experienced by the remaining electrons. This increased Coulomb attraction causes the valence atomic orbitals to collapse toward the nucleus. To describe such collapse, the basis set must have significant radial flexibility; angular flexibility is less important. For this reason, one should use at least a triple- $\zeta$ (or better) basis. The collapse of the core orbitals has even a larger energetic effect because of the large contribution of core electrons to the total electronic energy. According to Slater's rules [43], the shielding effect of one $1 s$ electron on the other $1 s$ electron is roughly 0.3 protons. This core collapse has huge energetic consequences; thus, it is essential to describe it well to obtain accurate results. The basis, therefore, should include a sufficient number of the core functions. This can be achieved by choosing polarized-core Dunning's sets (cc-pCVXZ) or by decontracting the core functions, such as the "6-" core function in the split-valence Pople bases, as was done in Refs. $[16,29,44]$. Core-correlation effects are considerably smaller (in energy) than these "radial collapse" effects and, for that reason, one may expect core-correlated basis sets to be less effective than core-decontracted ones [16]. To verify whether this expectation holds when using a high-level correlated method, we consider series of the original contracted Dunning and Pople basis sets of a triple- $\zeta$ quality and above and their partially or fully decontracted variants. The full description of the basis sets is given below.

\section{A. Computational Details}

All calculations were carried out using the Q-Chem electronic structure program[45, 46]. We employ the fc-CVS-EOM-IP-CCSD method[27] in which the target ionized states are described by the following ansatz:

$$
\Psi^{N-1}=\left(R_{1}+R_{2}\right) e^{T_{1}+T_{2}} \Phi_{0}(N)
$$


where $\Phi_{0}(N)$ denotes the reference determinant of an $N$-electron system, the singles and doubles excitation operators $T_{1}$ and $T_{2}$ contain the amplitudes for the reference state obtained by solving CCSD equations. The excitation operators $R_{1}$ and $R_{2}$ contain the EOM amplitudes obtained solving an EOM eigenproblem. While $T_{1}$ and $T_{2}$ are particle- and spin-conserving operators, the EOM-IP operators are of an ionizing type:

$$
R_{1}=\sum_{i} r_{i} i ; \quad R_{2}=\frac{1}{2} \sum_{i j a} r_{i j}^{a} a^{\dagger} j i
$$

Following the standard notation, indices $i, j, k, \ldots$ denote occupied orbitals and $a, b, c, \ldots$ denote virtual orbitals, as defined by the choice of the reference determinant $\Phi_{0}$. In fc-CVSEOM-IP-CCSD, the core electrons are frozen at the CCSD step (i.e., respective amplitudes in $T_{1}$ and $T_{2}$ are zero) and the EOM amplitudes should involve at least one core orbital, as prescribed by the CVS scheme.

The definition of the core in our CVS scheme depends on the edge[27]: the edge of interest and all lower edges are frozen while all higher edges are active. In this study we focus on the molecules containing first- and second-row elements (C,N,O, and H). Thus, in calculations of the carbon edge, the standard definition of the frozen core is used: all $1 s$ orbitals of the second-row atoms are frozen. In calculations of the nitrogen edge, only oxygen and nitrogen $1 s$ orbitals are frozen while carbon's $1 s$ orbitals are active. Likewise, in calculations of the oxygen edge, only $1 s_{O}$ orbitals are frozen and the rest of the core orbitals are active.

Our benchmark set comprises two simple diatomics, carbon monoxide (CO) and dinitrogen $\left(\mathrm{N}_{2}\right)$, three hydrides (water, ammonia, methane), and two polyatomic molecules, acrolein and glycine. This set allows us to investigate basis set effects for carbon, nitrogen, and oxygen edges, including molecules with several atoms of the same type and molecules with more than one edge.

The calculations for dinitrogen and carbon monoxide were carried out at the experimental geometries taken from Ref. [47] $\left(R_{N N}=1.097685 \AA\right.$ and $\left.R_{C O}=1.128323 \AA\right)$. The hydrides' structures were taken from Ref. [25], where they were optimized with RI-MP2/cc-pVTZ. For acrolein (Fig. 1), we used an MP2/cc-pVTZ optimized structure. Glycine calculations were performed for the canonical isomer (the main form of the gas-phase glycine) using the RI-MP2/cc-pVTZ optimized structure taken from Ref. [25] (Fig. 2).

We used Q-Chem's default convergence thresholds, except for the EOM amplitudes for 


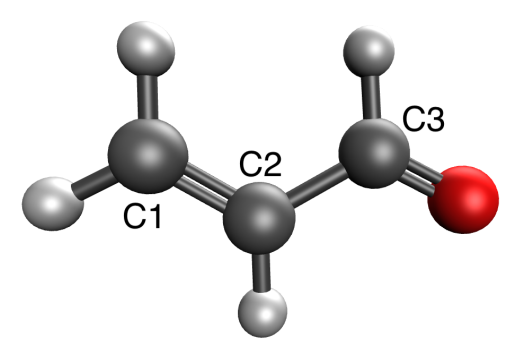

FIG. 1: Acrolein structure with atom labels.

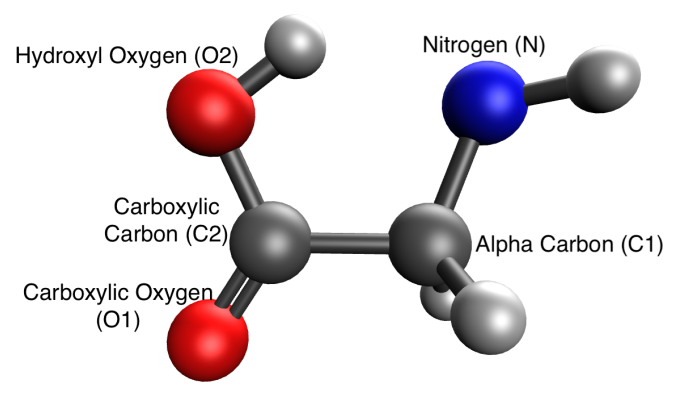

FIG. 2: Glycine structure (canonical isomer) with atom labels.

which a tighter threshold was used. SCF convergence was $10^{-8}$, CCSD convergence was $10^{-6}$, and the Davidson convergence was $10^{-7}$. In single-precision calculations (cf Sec. III E), CCSD convergence thresholds were $10^{-4}$ for amplitudes and $10^{-5}$ for energies.

The basis sets were decontracted manually and inputed as user-specified bases. For each basis, we considered two decontracted versions: one in which only the core orbitals were decontracted (this converts one core function from the $6-311+\mathrm{G}(3 \mathrm{df})$ basis set into six variationally independent functions) and one in which all functions were uncontracted. Using $6-311+\mathrm{G}(3 \mathrm{df})$ as an example, the latter procedure amounts to converting a triple- $\zeta$ basis into a $5-\zeta$ one. The redundant basis functions, which appear in decontracted Dunning's sets, were removed from the calculations.

Table I collects the basis sets used in this study, their contraction schemes, and the number of basis functions per atom for the second row elements. It also introduces shorthand notations for the uncontracted bases. We used pure angular momentum functions $(5 d$, $7 f, 9 h, 11 i, \ldots)$ for all bases. For Dunning's bases, we used the versions with optimized contraction, as implemented in Q-Chem. The aug-cc-pCV5Z and aug-cc-pV6Z bases were taken form the Basis Set Exchange database[48], without optimizing the general contractions (numeric tests indicated that using the variants of these bases with optimized general 
contractions leads to essentially the same results).

TABLE I: Basis sets, contraction schemes, and the number of functions per atom ${ }^{a}$.

\begin{tabular}{|c|c|c|c|}
\hline Basis & Contraction level & Contraction scheme & \#b.f. \\
\hline $6-311+\mathrm{G}(2 \mathrm{df})$ & Original & $(12 \mathrm{~s} 6 \mathrm{p} 2 \mathrm{~d} 1 \mathrm{f}) /[5 \mathrm{~s} 4 \mathrm{p} 2 \mathrm{~d} 1 \mathrm{f}]$ & 34 \\
\hline $\mathrm{uC}-6-311+\mathrm{G}(2 \mathrm{df})$ & Core-uncontracted & $(12 \mathrm{~s} 6 \mathrm{p} 2 \mathrm{~d} 1 \mathrm{f}) /[10 \mathrm{~s} 4 \mathrm{p} 2 \mathrm{~d} 1 \mathrm{f}]$ & 39 \\
\hline $\mathrm{u}-6-311+\mathrm{G}(2 \mathrm{df})$ & Fully uncontracted & $(12 \mathrm{~s} 6 \mathrm{p} 2 \mathrm{~d} 1 \mathrm{f}) /[12 \mathrm{~s} 6 \mathrm{p} 2 \mathrm{~d} 1 \mathrm{f}]$ & 47 \\
\hline $6-311+\mathrm{G}(3 \mathrm{df})$ & Original & $(12 \mathrm{~s} 6 \mathrm{p} 3 \mathrm{~d} 1 \mathrm{f}) /[5 \mathrm{~s} 4 \mathrm{p} 3 \mathrm{~d} 1 \mathrm{f}]$ & 39 \\
\hline $\mathrm{uC}-6-311+\mathrm{G}(3 \mathrm{df})$ & Core-uncontracted & $(12 \mathrm{~s} 6 \mathrm{p} 3 \mathrm{~d} 1 \mathrm{f}) /[10 \mathrm{~s} 4 \mathrm{p} 3 \mathrm{~d} 1 \mathrm{f}]$ & 44 \\
\hline $\mathrm{u}-6-311+\mathrm{G}(3 \mathrm{df})$ & Fully uncontracted & $(12 \mathrm{~s} 6 \mathrm{p} 3 \mathrm{~d} 1 \mathrm{f}) /[12 \mathrm{~s} 6 \mathrm{p} 3 \mathrm{~d} 1 \mathrm{f}]$ & 52 \\
\hline aug-cc-pVTZ & Original & $(11 \mathrm{~s} 6 \mathrm{p} 3 \mathrm{~d} 2 \mathrm{f}) /[5 \mathrm{~s} 4 \mathrm{p} 3 \mathrm{~d} 2 \mathrm{f}]$ & 46 \\
\hline uC-aug-cc-pVTZ & Core-uncontracted & $(11 \mathrm{~s} 6 \mathrm{p} 3 \mathrm{~d} 2 \mathrm{f}) /[11 \mathrm{~s} 4 \mathrm{p} 3 \mathrm{~d} 2 \mathrm{f}]$ & 52 \\
\hline u-aug-cc-pVTZ & Fully uncontracted & $(11 \mathrm{~s} 6 \mathrm{p} 3 \mathrm{~d} 2 \mathrm{f}) /[11 \mathrm{~s} 6 \mathrm{p} 3 \mathrm{~d} 2 \mathrm{f}]$ & 58 \\
\hline aug-cc-pVQZ & Original & $(13 \mathrm{~s} 7 \mathrm{p} 4 \mathrm{~d} 3 \mathrm{f}) /[6 \mathrm{~s} 5 \mathrm{p} 4 \mathrm{~d} 3 \mathrm{f} 2 \mathrm{~g}]$ & 80 \\
\hline uC-aug-cc-pVQZ & Core-uncontracted & $(13 \mathrm{~s} 7 \mathrm{p} 4 \mathrm{~d} 3 \mathrm{f} 2 \mathrm{~g}) /[13 \mathrm{~s} 5 \mathrm{p} 4 \mathrm{~d} 3 \mathrm{f} 2 \mathrm{~g}]$ & 87 \\
\hline u-aug-cc-pVQZ & Fully uncontracted & $(13 \mathrm{~s} 7 \mathrm{p} 4 \mathrm{~d} 3 \mathrm{f} 2 \mathrm{~g}) /[13 \mathrm{~s} 7 \mathrm{p} 4 \mathrm{~d} 3 \mathrm{f} 2 \mathrm{~g}]$ & 93 \\
\hline aug-cc-pV5Z & Original & (15s9p5d4f3g2h)/[7s6p5d4f3g2h] & 127 \\
\hline uC-aug-cc-pV5Z & Core-uncontracted & (15s9p5d4f3g2h)/[15s6p5d4f3g2h] & 135 \\
\hline u-aug-cc-pV5Z & Fully uncontracted & $(15 \mathrm{~s} 9 \mathrm{p} 5 \mathrm{~d} 4 \mathrm{f} 3 \mathrm{~g} 2 \mathrm{~h}) /[15 \mathrm{~s} 9 \mathrm{p} 5 \mathrm{~d} 4 \mathrm{f} 3 \mathrm{~g} 2 \mathrm{~h}]$ & 144 \\
\hline aug-cc-pV6Z & Original & (17s11p6d5f4g3h2i)/[8s7p6d5f4g3h2i] & 189 \\
\hline uC-aug-cc-pV6Z & Core-uncontracted & $(17 \mathrm{~s} 11 \mathrm{p} 6 \mathrm{~d} 5 \mathrm{ff} 4 \mathrm{~g} 3 \mathrm{~h} 2 \mathrm{i}) /[17 \mathrm{~s} 7 \mathrm{p} 6 \mathrm{~d} 5 \mathrm{f} 4 \mathrm{~g} 3 \mathrm{~h} 2 \mathrm{i}]$ & 198 \\
\hline u-aug-cc-pV6Z & Fully uncontracted & $(17 \mathrm{~s} 11 \mathrm{p} 6 \mathrm{~d} 5 \mathrm{f} 4 \mathrm{~g} 3 \mathrm{~h} 2 \mathrm{i}) /[17 \mathrm{~s} 11 \mathrm{p} 6 \mathrm{~d} 5 \mathrm{f} 4 \mathrm{~g} 3 \mathrm{~h} 2 \mathrm{i}]$ & 210 \\
\hline aug-cc-pCV5Z & Original & (19s13p8d6f4g2h)/[11s10p8d6f4g2h] & 181 \\
\hline uC-aug-cc-pCV5Z & Core-uncontracted & $(19 \mathrm{~s} 13 \mathrm{p} 8 \mathrm{~d} 6 \mathrm{f} 4 \mathrm{~g} 2 \mathrm{~h}) /[19 \mathrm{~s} 10 \mathrm{p} 8 \mathrm{~d} 6 \mathrm{f} 4 \mathrm{~g} 2 \mathrm{~h}]$ & 189 \\
\hline u-aug-cc-pCV5Z & Fully uncontracted & $(19 \mathrm{~s} 13 \mathrm{p} 8 \mathrm{~d} 6 \mathrm{f} 4 \mathrm{~g} 2 \mathrm{~h}) /[19 \mathrm{~s} 13 \mathrm{p} 8 \mathrm{~d} 6 \mathrm{f} 4 \mathrm{~g} 2 \mathrm{~h}]$ & 198 \\
\hline
\end{tabular}




\section{RESULTS AND DISCUSSION}

\section{A. Nitrogen molecule, $\mathbf{N}_{2}$}

The results for $\mathrm{N}_{2}$ are collected in Table II and shown graphically in Fig. 3. Table II shows total CCSD energy of the neutral reference state and two core IEs, corresponding to ionization from $\sigma_{u}(1 s)$ (lower value, IE1) and $\sigma_{g}(1 s)$ (higher value, IE2) orbitals. The total energies show anticipated trends: they decrease upon uncontraction and the magnitude of the decrease is larger when the valence orbitals are uncontracted than when only the core orbitals are uncontracted. The magnitude of this decrease is larger for Pople's bases than for Dunning's bases, which is also expected because the relative increase in the number of basis functions is larger for Pople's bases.

As noted in the previous EOM-CC benchmark study[35], the IEs decrease monotonously in the series of contracted basis sets of increasing size. The IEs also generally decrease upon uncontraction. This is a manifestation of core-relaxation effects, which lower the energy of the target ionized state. In contrast to the total energies, the drop in IE is always larger when the core orbitals are uncontracted than when the valence orbitals are uncontracted. The magnitude of the change is larger for smaller bases than for larger bases because the increase in the basis size is larger for the smaller bases. We also observe that the changes are rather small when polarized-core basis is used, because these bases already afford sufficient flexibility in describing core electrons.

The results in Table II show that the IEs reach the basis-set limit within $0.01 \mathrm{eV}$ for augcc-pCV5Z (and the respective uncontracted variants), uC-aug-cc-pV6Z/u-aug-cc-pV6Z, and u-aug-cc-pV5Z. The smallest among these bases is aug-cc-pCV5Z, which is not surprising, as this basis has more functions optimized for the core description (although they are optimized for describing the correlation of the core electrons in the ground state).

The energy gap between the two core states converges much faster with respect to the basis set than the absolute values of IEs, owing to error cancellation. For example, the difference between the smallest basis $(6-311+\mathrm{G}(2 \mathrm{df}))$ and the basis-set limit is less than $0.001 \mathrm{eV}$.

Fig. 3 shows the lower core IE $\left(\sigma_{u}\right)$ for all basis sets as a function of the number of basis functions, which clearly indicates the effectiveness of different bases in describing core IEs. 
While it is not surprising that larger bases perform better than smaller bases, as illustrated by the smooth trend of the red curve (original contracted bases sets), the difference between contracted and core-uncontracted bases is remarkable. For example, uC-aug-cc-pVQZ gives better result than aug-cc-pV5Z, despite being 1.5 times more compact. The performance of core and fully uncontracted Pople's bases is even more impressive - uncontracted u-6$311+\mathrm{G}(3 \mathrm{df})$ (with 52 functions per atom) delivers the same result as uC-aug-cc-pVQZ (87 functions per atom). Overall, the values with fully uncontracted $u-6-311+G(3 \mathrm{df})$ are within $0.06 \mathrm{eV}$ from the basis set limit (u-aug-cc-pV5Z). Adding a second set of diffuse functions to uC-6-311+G(3df) lowers the IE by $0.002 \mathrm{eV}$ (see Table II). Somewhat unexpectedly, the uncontracted aug-cc-pVTZ bases yield noticeably larger errors relative to the basis set limit than more compact uncontracted u-/uC-6-311+G(3df) bases.

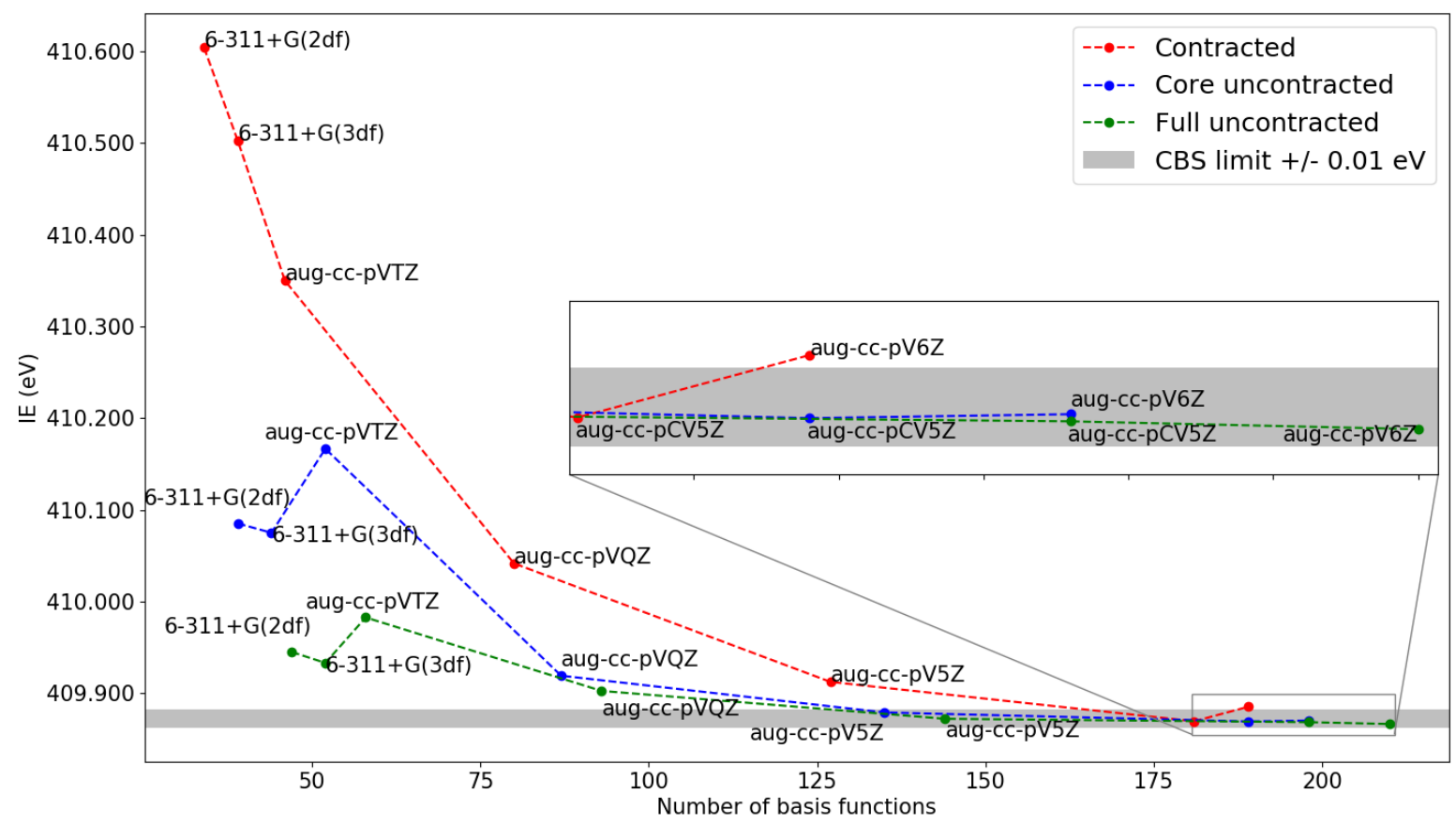

FIG. 3: Core IEs for $\mathrm{N}_{2}$ versus the number of basis functions per atom. Gray shaded area marks $\pm 0.01 \mathrm{eV}$ interval around the basis-set limit (u-aug-cc-pV5Z).

\section{B. Carbon monoxide, CO}

The results for CO are collected in Tables III and IV and shown graphically in Figs. 4 and 5. The experimental values were taken from Ref. [49]. Overall, the trends for both 
TABLE II: Core IEs for $\mathrm{N}_{2}$, fc-CVS-EOM-IP-CCSD with various basis sets.

\begin{tabular}{|c|c|c|c|}
\hline Basis & CCSD energy $^{a}$ (a.u.) & $\mathrm{IE}^{b}(\mathrm{eV})$ & $\mathrm{IE} 2(\mathrm{eV}) \Delta \mathrm{IE}(\mathrm{eV})$ \\
\hline $6-311+\mathrm{G}(2 \mathrm{df})$ & -109.350207 & 410.6041 & $410.4996 \quad 0.1045$ \\
\hline $\mathrm{uC}-6-311+\mathrm{G}(2 \mathrm{df})$ & -109.353623 & 410.0853 & 409.98020 .1051 \\
\hline $\mathrm{u}-6-311+\mathrm{G}(2 \mathrm{df})$ & -109.357797 & 409.9451 & 409.84020 .1049 \\
\hline $6-311+\mathrm{G}(3 \mathrm{df})$ & -109.354688 & 410.5026 & 410.39810 .1045 \\
\hline $\mathrm{uC}-6-311+\mathrm{G}(3 \mathrm{df})$ & -109.357388 & 410.0750 & $409.9701 \quad 0.1049$ \\
\hline $\mathrm{u}-6-311+\mathrm{G}(3 \mathrm{df})$ & -109.361379 & 409.9331 & 409.82820 .1049 \\
\hline $6-311(2+) \mathrm{G}(3 \mathrm{df})$ & -109.354828 & 410.4998 & 410.39530 .1045 \\
\hline $\mathrm{uC}-6-311(2+) \mathrm{G}(3 \mathrm{df})$ & -109.357517 & 410.0725 & $409.9676 \quad 0.1049$ \\
\hline $\mathrm{u}-6-311(2+) \mathrm{G}(3 \mathrm{df})$ & -109.361498 & 409.9315 & 409.82660 .1049 \\
\hline aug-cc-pVTZ & -109.361574 & 410.3500 & 410.24540 .1046 \\
\hline uC-aug-cc-pVTZ & -109.363030 & 410.1667 & $410.0620 \quad 0.1047$ \\
\hline u-aug-cc-pVTZ & -109.366680 & 409.9827 & 409.87810 .1046 \\
\hline aug-cc-pVQZ & -109.386793 & 410.0417 & 409.93700 .1047 \\
\hline uC-aug-cc-pVQZ & -109.387264 & 409.9192 & 409.81430 .1049 \\
\hline u-aug-cc-pVQZ & -109.387920 & 409.4026 & $409.7976 \quad 0.1050$ \\
\hline aug-cc-pV5Z & -109.394586 & 409.9124 & 409.80730 .1051 \\
\hline uC-aug-cc-pV5Z & -109.394648 & 409.8791 & $409.7740 \quad 0.1051$ \\
\hline u-aug-cc-pV5Z & -109.395016 & 409.8722 & 409.76710 .1051 \\
\hline aug-cc-pV6Z & -109.397296 & 409.8853 & 409.78020 .1051 \\
\hline uC-aug-cc-pV6Z & -109.397313 & 409.8703 & 409.76520 .1051 \\
\hline u-aug-cc-pV6Z & -109.397514 & 409.8665 & $409.7614 \quad 0.1051$ \\
\hline aug-cc-pCV5Z & -109.395330 & 409.8695 & $409.7644 \quad 0.1051$ \\
\hline uC-aug-cc-pCV5Z & -109.395322 & 409.8693 & 409.76420 .1051 \\
\hline u-aug-cc-pCV5Z & -109.395413 & 409.8685 & 409.76340 .1051 \\
\hline
\end{tabular}

${ }^{a}$ Total energy for the neutral reference state. ${ }^{b}$ Experimental $\mathrm{IE}_{\sigma_{u}}=409.9 \mathrm{eV}$ is taken from Ref. [25].

edges follow very closely the trends observed for $\mathrm{N}_{2}$, reinforcing the main finding - impressive effectiveness of uncontracted Pople's bases in describing the core IEs. For the carbon edge, $\mathrm{u}-6-311+\mathrm{G}(3 \mathrm{df})$ is within $0.01 \mathrm{eV}$ from the basis-set limit (u-aug-cc-pV5Z), whereas for the oxygen edge the difference is slightly larger $(0.1 \mathrm{eV})$.

Using this molecule with two edges as an example, we tested the protocol of using different bases for active and inactive edges, e.g., using uncontracted bases for both $\mathrm{C}$ and $\mathrm{O}$ or using an uncontracted basis for the active edge and an original, contracted basis for the inactive edge. The results show that the difference between the two schemes is small (except for 
the smallest basis, 6-311+G(2df)), suggesting an effective compromise for calculations of polyatomic heteronuclear molecules.
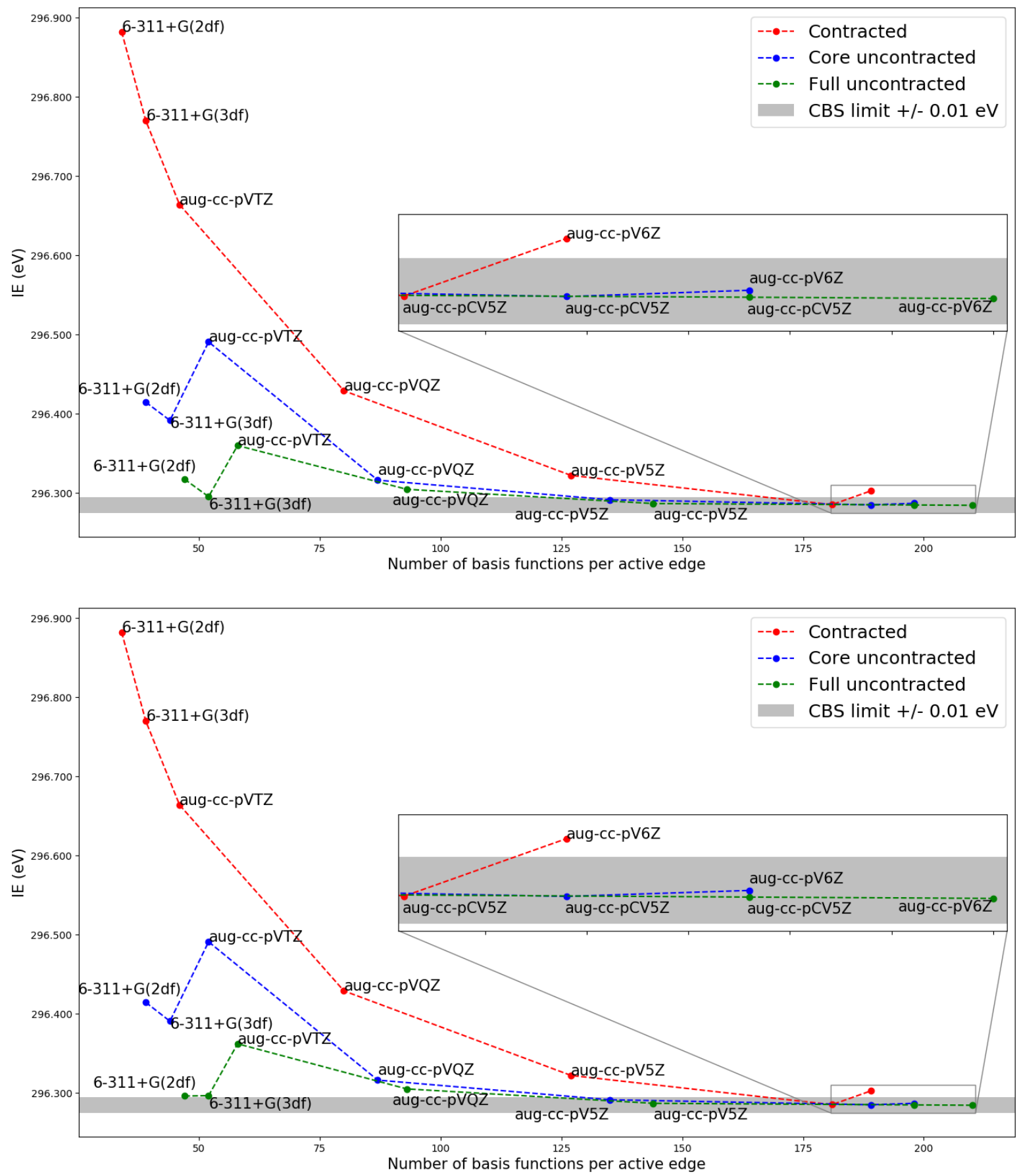

FIG. 4: CO, carbon edge IEs. Top: Only carbon basis is uncontracted, original matching basis is used for oxygen. Bottom: Both carbon and oxygen bases are uncontracted. Gray shaded area marks the $\pm 0.01 \mathrm{eV}$ interval around the basis-set limit (u-aug-ccp-pV5Z). 

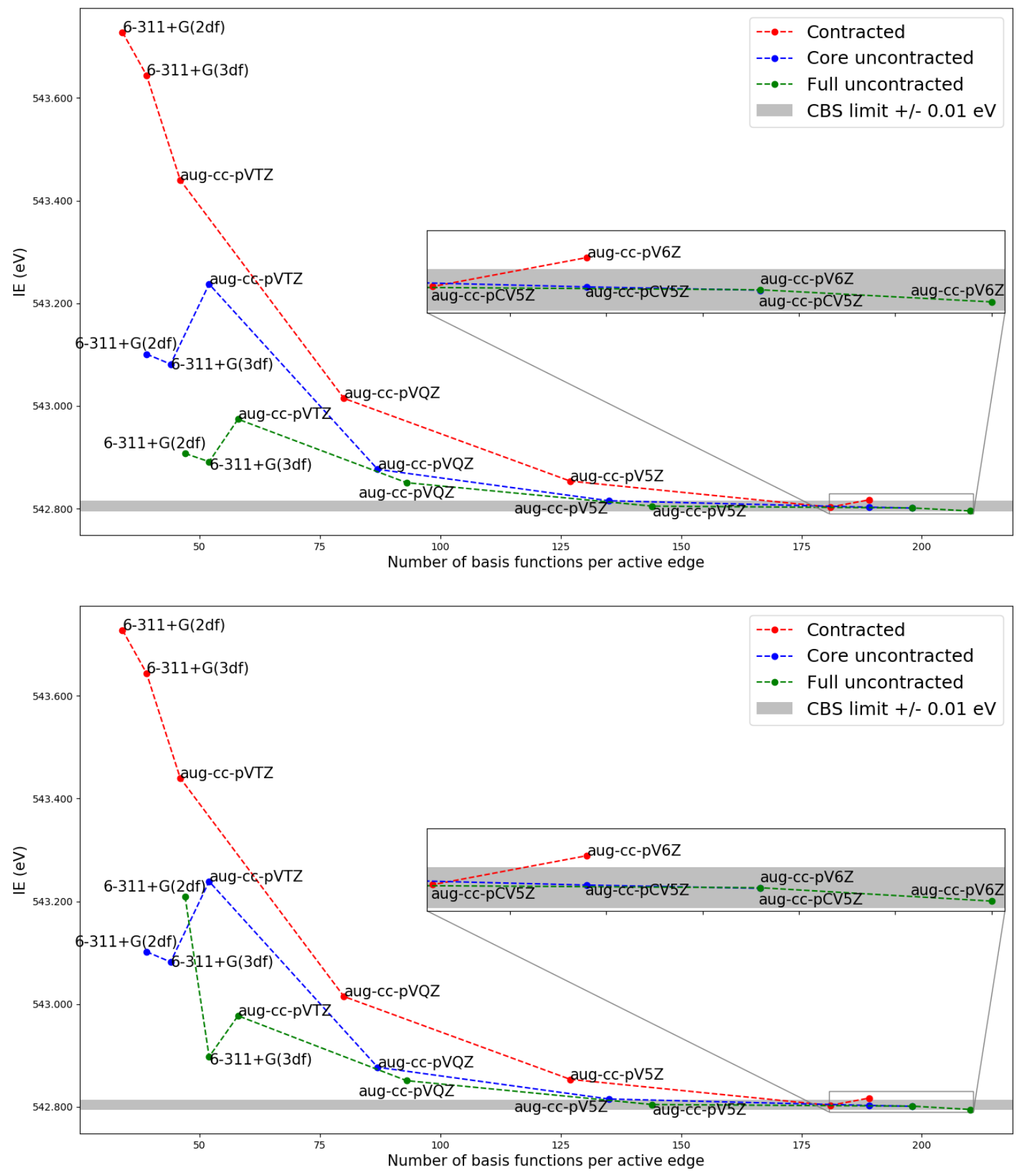

FIG. 5: CO, oxygen edge IEs. Top: Only oxygen basis is uncontracted, original matching basis is used for carbon. Bottom: Both carbon and oxygen bases are uncontracted. Gray shaded area marks the $\pm 0.01 \mathrm{eV}$ interval around the basis-set limit (u-aug-ccp-pV5Z). 
TABLE III: CO, carbon edge. Total and ionization energies; fc-CVS-EOM-IP-CCSD with various basis sets.

\begin{tabular}{|c|c|c|c|}
\hline Basis on $\mathrm{C}$ & Basis on $\mathrm{O}$ & CCSD energy $^{a}$ (a.u.) & $\mathrm{IE}^{b}(\mathrm{eV})$ \\
\hline \multirow{2}{*}{$6-311+\mathrm{G}(2 \mathrm{df})$} & $6-311+\mathrm{G}(2 \mathrm{df})$ & -113.133212 & 296.8822 \\
\hline & $6-311+\mathrm{G}(2 \mathrm{df})$ & -113.134520 & 296.4147 \\
\hline $\mathrm{uC}-6-311+\mathrm{G}(2 \mathrm{df})$ & $\mathrm{uC}-6-311+\mathrm{G}(2 \mathrm{df})$ & -113.142902 & 296.3908 \\
\hline \multirow{2}{*}{$\mathrm{u}-6-311+\mathrm{G}(2 \mathrm{df})$} & $6-311+\mathrm{G}(2 \mathrm{df})$ & -113.136044 & 296.3176 \\
\hline & $\mathrm{u}-6-311+\mathrm{G}(2 \mathrm{df})$ & -113.122722 & 296.2963 \\
\hline \multirow{3}{*}{$\begin{array}{l}6-311+\mathrm{G}(3 \mathrm{df}) \\
\mathrm{uC}-6-311+\mathrm{G}(3 \mathrm{df})\end{array}$} & $6-311+\mathrm{G}(3 \mathrm{df})$ & -113.139488 & 296.7699 \\
\hline & $6-311+\mathrm{G}(3 \mathrm{df})$ & -113.140761 & 296.3917 \\
\hline & $\mathrm{uC}-6-311+\mathrm{G}(3 \mathrm{df})$ & -113.142902 & 296.3908 \\
\hline \multirow{2}{*}{$\mathrm{u}-6-311+\mathrm{G}(3 \mathrm{df})$} & $6-311+\mathrm{G}(3 \mathrm{df})$ & -113.142200 & 296.2955 \\
\hline & $\mathrm{u}-6-311+\mathrm{G}(3 \mathrm{df})$ & -113.147177 & 296.2968 \\
\hline \multirow{3}{*}{$\begin{array}{l}\text { aug-cc-pVTZ } \\
\text { uC-aug-cc-pVTZ }\end{array}$} & aug-cc-pVTZ & -113.1445197 & 296.6638 \\
\hline & aug-cc-pVTZ & -113.144989 & 296.4907 \\
\hline & uC-aug-cc-pVTZ & -113.145565 & 296.4911 \\
\hline \multirow{2}{*}{ u-aug-cc-pVTZ } & aug-cc-pVTZ & -113.146335 & 296.3600 \\
\hline & u-aug-cc-pVTZ & -113.149699 & 296.3623 \\
\hline \multirow{3}{*}{$\begin{array}{l}\text { aug-cc-pVQZ } \\
\text { uC-aug-cc-pVQZ }\end{array}$} & aug-cc-pVQZ & -113.171609 & 296.4291 \\
\hline & aug-cc-pVQZ & -113.171748 & 296.3163 \\
\hline & uC-aug-cc-pVQZ & -113.172132 & 296.3164 \\
\hline \multirow{2}{*}{ u-aug-cc-pVQZ } & aug-cc-pVQZ & -113.171948 & 296.3048 \\
\hline & u-aug-cc-pVQZ & -113.172888 & 296.3053 \\
\hline \multirow[t]{2}{*}{ aug-cc-pV5Z } & aug-cc-pV5Z & -113.180060 & 296.3223 \\
\hline & aug-cc-pV5Z & -113.180078 & 296.2916 \\
\hline
\end{tabular}




\begin{tabular}{llll} 
& uC-aug-cc-pV5Z & -113.180128 & 296.2916 \\
\cline { 2 - 4 } u-aug-cc-pV5Z & aug-cc-pV5Z & -113.180193 & 296.2868 \\
& u-aug-cc-pV5Z & -113.180538 & 296.2871 \\
\cline { 2 - 4 } aug-cc-pV6Z & aug-cc-pV6Z & -113.183007 & 296.3027 \\
\cline { 2 - 4 } uC-aug-cc-pV6Z & aug-cc-pV5Z & -113.183014 & 296.2871 \\
& uC-aug-cc-pV6Z & -113.183029 & 296.2871 \\
\cline { 2 - 4 } u-aug-cc-pV6Z & aug-cc-pV6Z & -113.183076 & 296.2846 \\
\hline aug-cc-pCV5Z & aug-aug-cc-pV6Z & -113.183257 & 296.2847 \\
\cline { 2 - 4 } uC-aug-cc-pCV5Z & aug-cc-pCV5Z & -113.180916 & 296.2854 \\
\cline { 2 - 4 } & uC-aug-cc-pCV5Z & -113.180917 & 296.2853 \\
\cline { 2 - 4 } u-aug-cc-pCV5Z & aug-cc-pCV5Z & -113.180928 & 296.2850 \\
& u-aug-cc-pCV5Z & -113.181004 & 296.2851 \\
\hline
\end{tabular}

${ }^{a}$ Total energy for the neutral reference state. ${ }^{b}$ Experimental IE for the $\mathrm{C}$ edge $296.2 \mathrm{eV}$ is taken from Ref. [49] 
TABLE IV: CO, oxygen edge. Total and ionization energies; fc-CVS-EOM-IP-CCSD with various basis sets.

\begin{tabular}{|c|c|c|c|}
\hline Basis on $\mathrm{O}$ & Basis on $\mathrm{C}$ & CCSD energy $^{a}$ (a.u.) & $\mathrm{IE}^{b}(\mathrm{eV})$ \\
\hline $6-311+\mathrm{G}(2 \mathrm{df})$ & $6-311+\mathrm{G}(2 \mathrm{df})$ & -113.153403 & 543.7282 \\
\hline \multirow{2}{*}{$\mathrm{uC}-6-311+\mathrm{G}(2 \mathrm{df})$} & $6-311+\mathrm{G}(2 \mathrm{df})$ & -113.156050 & 543.1010 \\
\hline & $\mathrm{uC}-6-311+\mathrm{G}(2 \mathrm{df})$ & -113.159353 & 543.1019 \\
\hline \multirow{2}{*}{$\mathrm{u}-6-311+\mathrm{G}(2 \mathrm{df})$} & $6-311+\mathrm{G}(2 \mathrm{df})$ & -113.159201 & 542.9073 \\
\hline & $\mathrm{u}-6-311+\mathrm{G}(2 \mathrm{df})$ & -113.167966 & 543.2097 \\
\hline $6-311+\mathrm{G}(3 \mathrm{df})$ & $6-311+\mathrm{G}(3 \mathrm{df})$ & -113.160756 & 543.6443 \\
\hline \multirow{2}{*}{$\mathrm{uC}-6-311+\mathrm{G}(3 \mathrm{df})$} & $6-311+\mathrm{G}(3 \mathrm{df})$ & -113.163070 & 543.0814 \\
\hline & $\mathrm{uC}-6-311+\mathrm{G}(3 \mathrm{df})$ & -113.166218 & 543.0820 \\
\hline \multirow{2}{*}{$\mathrm{u}-6-311+\mathrm{G}(3 \mathrm{df})$} & $6-311+\mathrm{G}(3 \mathrm{df})$ & -113.166134 & 542.8913 \\
\hline & $\mathrm{u}-6-311+\mathrm{G}(3 \mathrm{df})$ & -113.193741 & 542.8975 \\
\hline \multirow[t]{2}{*}{ aug-cc-pVTZ } & aug-cc-pVTZ & -113.159233 & 543.4400 \\
\hline & aug-cc-pVTZ & -113.159820 & 543.2373 \\
\hline uC-aug-cc-pVTZ & uC-aug-cc-pVTZ & -113.166897 & 543.2388 \\
\hline \multirow{2}{*}{ u-aug-cc-pVTZ } & aug-cc-pVTZ & -113.162900 & 542.9741 \\
\hline & u-aug-cc-pVTZ & -113.194170 & 542.9772 \\
\hline \multirow[t]{2}{*}{ aug-cc-pVQZ } & aug-cc-pVQZ & -113.199349 & 543.0147 \\
\hline & aug-cc-pVQZ & -113.199742 & 542.8764 \\
\hline uC-aug-cc-pVQZ & uC-aug-cc-pVQZ & -113.201736 & 542.8770 \\
\hline \multirow{2}{*}{ u-aug-cc-pVQZ } & aug-cc-pVQZ & -113.200312 & 542.8504 \\
\hline & u-aug-cc-pVQZ & -113.220245 & 542.8509 \\
\hline aug-cc-pV5Z & aug-cc-pV5Z & -113.213669 & 542.8534 \\
\hline \multirow{3}{*}{ uC-aug-cc-pV5Z } & aug-cc-pV5Z & -113.213736 & 542.8152 \\
\hline & uC-aug-cc-pV5Z & -113.214943 & 542.8152 \\
\hline & aug-cc-pV5Z & -113.214077 & 542.8047 \\
\hline
\end{tabular}




\begin{tabular}{llll} 
& \multicolumn{1}{c}{$\mathrm{u}$-aug-cc-pV5Z } & -113.229862 & 542.8044 \\
\cline { 2 - 4 } aug-cc-pV6Z & aug-cc-pV6Z & -113.222164 & 542.8169 \\
\cline { 2 - 4 } uC-aug-cc-pV6Z & aug-cc-pV5Z & -113.222180 & 542.8010 \\
& uC-aug-cc-pV6Z & -113.223047 & 542.8010 \\
\cline { 2 - 4 } u-aug-cc-pV6Z & aug-cc-pV6Z & -113.222351 & 542.7953 \\
\hline aug-cc-pCV5Z & a-aug-cc-pV6Z & -113.233567 & 542.7948 \\
\cline { 2 - 4 } uC-aug-cc-pCV5Z & aug-cc-pCV5Z & -113.235385 & 542.8026 \\
\cline { 2 - 4 } u-aug-cc-pCV5Z & aug-aug-cc-pCV5Z & -113.235389 & 542.8026 \\
\cline { 2 - 4 } & u-aug-cc-pCV5Z & -113.235509 & 542.8028 \\
\hline
\end{tabular}

${ }^{a}$ Total energy for the neutral reference state. ${ }^{b}$ Experimental IE for the O edge IE 542.5 $\mathrm{eV}$ is taken from [49] 


\section{Simple hydrides and the effect of basis on hydrogen}

Small hydrides, water, ammonia, and methane, represent 3 different edges in molecules with hydrogen atoms. We use this set to investigate the effect that the basis set on the $\mathrm{H}$ atoms has on the heavy atoms' core IEs. We compare our findings with those of the previous study[35]. Table V shows the results computed with the Pople (3df) and Dunning basis sets on the heavy atom, combined with the matching contracted bases on hydrogen.

The results in Table V show that the differences between the smaller bases are similar to the results for $\mathrm{N}_{2}$ and $\mathrm{CO}$ and that the IEs converge from above to the basis-set limit. The basis-set limit results are slightly above the experimental values; the largest difference from the experiment is observed for carbon $(0.18 \mathrm{eV})$. This is similar to the findings in Ref. [35].

In contrast to the observation in Ref. [35], that the effect of the basis set beyond triple$\zeta$ is moderate $(0.1 \mathrm{eV}$ difference between the triple- $\zeta$ to quadruple- $\zeta$ results $)$, we observe somewhat larger effects for this set $(\sim 0.4 \mathrm{eV})$, as well as for the $\mathrm{N}_{2}$ and $\mathrm{CO}$ molecules discussed above. This difference is likely due to the slightly different treatment of core electrons in the ground-state optimization step in the CVS-EOM-CCSD and fc-CVS-EOMCCSD (we also note that the structures used in Ref. [35] are slightly different). However, going from quadruple to quintuple- $\zeta$, we observe a similar change of $<0.1 \mathrm{eV}$. Thus, the results of both studies indicate near-convergence to the basis-set limit at the quintuple- $\zeta$ basis. As the largest basis in the present calculations, we use uC-aug-cc-pV5Z (the IEs drop by $\sim 0.03 \mathrm{eV}$ upon uncontraction); below, we refer to these results as the basis-set limit.

Table VI collects the IEs computed with uC-aug-cc-pV5Z on the heavy atom and smaller bases on hydrogens. As expected, the effect of the hydrogen basis on the core IEs is not large. For example, using aug-cc-pVQZ or even aug-cc-pVTZ instead of aug-cc-pV5Z changes the IEs by less than 0.001 and $0.005 \mathrm{eV}$, respectively, while significantly reducing the number of basis functions. Thus, one may consider using a contracted triple- $\zeta$ basis (or even smaller) on hydrogens in calculations of larger molecules as a viable cost-saving strategy.

\section{Using mixed basis sets for molecules with multiple edges}

In this section we further investigate the idea of using mixed bases sets. We use the $\mathrm{CO}$ molecule and employ a larger basis (uC-aug-cc-pV5Z) on the active edge, and a smaller basis 
TABLE V: Core IEs for $\mathrm{H}_{2} \mathrm{O}, \mathrm{NH}_{3}$, and $\mathrm{CH}_{4}$.

\begin{tabular}{|c|c|c|c|c|c|}
\hline \multicolumn{2}{|c|}{ Basis on active edge Basis on $\mathrm{H}$} & \multirow{2}{*}{ \#b.f. (H) } & \multicolumn{3}{|c|}{ Core IE $(\mathrm{eV})$} \\
\hline & & & $\overline{\mathrm{H}_{2} \mathrm{O}}$ & $\mathrm{NH}_{3}$ & $\mathrm{CH}_{4}$ \\
\hline $6-311+\mathrm{G}(3 \mathrm{df})$ & $6-311 \mathrm{G}$ & 3 & 540.9000 & 406.3149 & 291.0717 \\
\hline $\mathrm{uC}-6-311+\mathrm{G}(3 \mathrm{df})$ & $6-311 G$ & 3 & 540.2500 & 405.8179 & 290.7117 \\
\hline aug-cc-pVTZ & aug-cc-pVTZ & 23 & 540.6570 & 406.0944 & 290.9248 \\
\hline uC-aug-cc-pVTZ & aug-cc-pVTZ & 23 & 540.4573 & 405.9 & 290.8383 \\
\hline aug-cc-pVQZ & aug-cc-pVQZ & 46 & 540.2110 & 405.7655 & 290.6862 \\
\hline uC-aug-cc-pVQZ & aug-cc-pVQZ & 46 & 540.0857 & 405.6690 & 290.6209 \\
\hline aug-cc-pV5Z & aug-cc-pV5Z & 80 & 540.0530 & 405.6505 & 290.6103 \\
\hline uC-aug-cc-pV5Z & aug-cc-pV5Z & 80 & 540.0162 & 405.6197 & 290.5845 \\
\hline \multicolumn{2}{|c|}{ Experimental core $\operatorname{IEs}^{a}(\mathrm{eV})$} & & 539.9 & 405.6 & 290.76 \\
\hline
\end{tabular}

TABLE VI: Core IEs for $\mathrm{H}_{2} \mathrm{O}, \mathrm{NH}_{3}$, and $\mathrm{CH}_{4}$ computed with mixed basis sets.

\begin{tabular}{|c|c|c|c|c|c|}
\hline \multirow{2}{*}{\multicolumn{2}{|c|}{ Basis on active edge Basis on $\mathrm{H}$}} & \multirow{2}{*}{ \#b.f. (H } & \multicolumn{2}{|c|}{ Core IE (eV) } & \multirow[b]{2}{*}{$\mathrm{CH}_{4}$} \\
\hline & & & $\mathrm{H}_{2} \mathrm{O}$ & $\mathrm{NH}_{3}$ & \\
\hline \multirow{7}{*}{ uC-aug-cc-pV5Z } & aug-cc-pV5Z & 80 & 540.016 & 405.619 & 290.5845 \\
\hline & u-aug-cc-pVQZ & 48 & 540.01 & 405.618 & 290.5843 \\
\hline & aug-cc-pVQZ & 46 & 540.01 & 405.618 & 290.5839 \\
\hline & u-aug-cc-pVTZ & 25 & 540.010 & 405.614 & 290.5805 \\
\hline & aug-cc-pVTZ & 23 & 540.010 & 405.613 & 290.5795 \\
\hline & aug-cc-pVDZ & 9 & 539.997 & 405.597 & 290.5617 \\
\hline & cc-pVDZ & 5 & 539.996 & 405.595 & 290.5621 \\
\hline
\end{tabular}

on the inactive edge. Table VII shows the results of these calculations. We observe that using a quadruple- $\zeta$ or even a triple- $\zeta$ basis on inactive edges leads to relatively small differences in IEs (less than $0.05 \mathrm{eV}$ ). However, the IEs no longer approach the basis-set limit from above. For example, the calculation with aug-cc-pVDZ on the inactive edge yields smaller IE than the calculation with aug-cc-pVQZ on the inactive edge, which indicates potential imbalance of such approach.

\section{E. Other cost-saving strategies}

In larger molecules, using uC-aug-cc-pV5Z on all heavy atoms quickly becomes prohibitively expensive. For example, for molecules with just 4 second row atoms, the total number of basis functions in uC-aug-cc-pV5Z exceeds 500. Aside the obvious choice of using a smaller set, the cost of the calculations can be reduced by using single-precision execution[51] and truncation of virtual space using frozen natural orbitals approach[52, 53]. 
TABLE VII: Core IEs in CO computed with mixed basis sets on carbon and oxygen edges ${ }^{a}$.

\begin{tabular}{llll} 
Basis on C & Basis on O & CCSD energy $^{b}$ (a.u.) $\mathrm{IE}^{a}(\mathrm{eV})$ \\
\hline & aug-cc-pVDZ & -113.118361 & 296.2136 \\
uC-aug-cc-pV5Z & aug-cc-pVTZ & -113.159656 & 296.2589 \\
& aug-cc-pVQZ & -113.174465 & 296.2832 \\
& aug-cc-pV5Z & -113.180916 & 296.2852 \\
\hline aug-cc-pVDZ & & -113.172430 & 542.7505 \\
aug-cc-pVTZ & uC-aug-cc-pV5Z & -113.193331 & 542.7850 \\
aug-cc-pVQZ & & -113.206596 & 542.8045 \\
aug-cc-pV5Z & & -113.235385 & 542.8026 \\
\hline
\end{tabular}

${ }^{a}$ The uC-aug-cc-pV5Z basis is used on active edges. ${ }^{b}$ Total energy for the neutral reference state.

Using single-precision execution limits the convergence thresholds. Thus, because in the benchmark study we desire tight convergence for the EOM energies, here we use single precision for the CCSD step only. Because CCSD is the scaling-determining step in the EOM-IP-CCSD calculations, using single precision leads to noticeable speedup. The results are shown in Table VIII. In agreement with previous benchmark[51], the effect of using single-precision at the CCSD step is negligible.

The FNO results are collected in Table IX. We use an occupation criterion to control the truncation of the virtual space (for example, the FNO threshold of $99.99 \%$ means that this much of the total natural occupation is recovered within the truncated orbital space). We observe that the errors due to virtual space truncation for a particular value of FNO threshold are similar for all three hydrides. The errors with FNO threshold of 99.99\%, which amounts to freezing $27-28 \%$ of the virtual orbitals, are around $0.06 \mathrm{eV}$. This relatively large value illustrates the importance of virtual orbitals in describing the relaxation effects due to core ionization.

Combining the FNO approximation with single precision at the CCSD level leads to noticeable reduction of computational time in methane and ammonia (about 7-fold speedup), while the effect in water was much smaller.

\section{F. Acrolein}

Acrolein (shown in Fig. 1) is an interesting model system with 3 chemically distinct carbon atoms: $\mathrm{C} 1$ is connected to 2 hydrogens and one carbon, $\mathrm{C} 2$ is connected to two carbons and one hydrogen, and C3 is connected to one hydrogen, one carbon, and one oxygen. 
TABLE VIII: Core IEs for $\mathrm{H}_{2} \mathrm{O}, \mathrm{NH}_{3}$, and $\mathrm{CH}_{4}$ computed with single and double precision $\mathrm{CCSD}^{a}$.

\begin{tabular}{|c|c|c|c|}
\hline Molecule & Precisiol & CCSD energy (a.u. $)^{b}$ & Core IE (eV) \\
\hline \multirow{2}{*}{$\mathrm{H}_{2} \mathrm{O}$} & Double & -76.35986226 & 540.0151 \\
\hline & Single & -76.35986228 & 540.0151 \\
\hline \multirow{2}{*}{$\mathrm{NH}_{3}$} & Double & -56.49013066 & 405.6189 \\
\hline & Single & -56.49013076 & 405.6189 \\
\hline \multirow{2}{*}{$\mathrm{CH}_{4}$} & Double & -40.44666824 & 290.5843 \\
\hline & Single & -40.44666822 & 290.5843 \\
\hline
\end{tabular}

${ }^{a}$ Active edge basis: uC-aug-cc-pV5Z, H basis: uC-aug-cc-pVQZ. ${ }^{b}$ Total energy for the neutral reference state. 8 decimal places are shown in order to demonstrate that the difference is only in the 8 th decimal place. CCSD convergence thresholds in single-precision calculation: $10^{-4}$ for amplitudes and $10^{-5}$ for energies.

TABLE IX: Core IEs for $\mathrm{H}_{2} \mathrm{O}, \mathrm{NH}_{3}$, and $\mathrm{CH}_{4}$ computed with the FNO-based truncation of the virtual space.

\begin{tabular}{|c|c|c|c|c|c|}
\hline Molecule $^{a}$ & FNO thresh ${ }^{b}$ & Act. virt. & Frzn. virt. & $\left.\right|_{\text {CCSD energy (a.u. })^{c}}$ & $\Delta \mathrm{IE}^{d}(\mathrm{eV})$ \\
\hline $\mathrm{H}_{2} \mathrm{O}$ & & 48 & 178 & -76.348978 & -0.6967 \\
\hline $\mathrm{NH}_{3}$ & 99.00 & 55 & 219 & -56.481735 & -0.5238 \\
\hline $\mathrm{CH}_{4}$ & & 65 & 257 & -40.440410 & -0.5280 \\
\hline $\mathrm{H}_{2} \mathrm{O}$ & & 115 & 111 & -76.358738 & -0.1541 \\
\hline $\mathrm{NH}_{3}$ & 99.90 & 135 & 139 & -56.489228 & -0.1781 \\
\hline $\mathrm{CH}_{4}$ & & 159 & 163 & -40.446014 & -0.1632 \\
\hline $\mathrm{H}_{2} \mathrm{O}$ & & 165 & 61 & -76.359781 & -0.0656 \\
\hline $\mathrm{NH}_{3}$ & 99.99 & 200 & 74 & -56.490056 & -0.0670 \\
\hline $\mathrm{CH}_{4}$ & & 233 & 89 & -40.446609 & -0.0614 \\
\hline
\end{tabular}

${ }^{a}$ Active edge basis: $\mathrm{uC}$-aug-cc-pV5Z, H basis: $\mathrm{uC}$-aug-cc-pVQZ. ${ }^{b}$ Population threshold: this fraction of total natural occupation is recovered by the active virtual orbitals. ${ }^{c}$ Total energy for the neutral reference state. ${ }^{d}$ IE shift relative to the full orbital space values in Table VIII.

We use this molecule to test how multiple IEs corresponding to the same edge are described with different bases and test whether our observations based on $\mathrm{CO}$ are transferable to a larger molecule. The available experimental results for the carbon edge, reported as shifts relative to $\mathrm{C} 1$, are from Ref. [54].

Table $\mathrm{X}$ and XI collect the results obtained using aug-cc-pV5Z and uC-aug-cc-pV5Z for the active edge. We observe that for both edges uncontracting the core in this basis leads 
TABLE X: Acrolein, core IEs for the oxygen edge.

\begin{tabular}{|c|c|c|c|c|}
\hline Basis on $\mathrm{C}$ & Basis on $\mathrm{O}$ & Basis on $\mathrm{H}$ & CCSD energy $^{a}$ (a.u) & $\mathrm{IE}(\mathrm{eV})$ \\
\hline & $6-311+\mathrm{G}(3 \mathrm{df})$ & & -191.605984 & 540.0669 \\
\hline \multirow[t]{2}{*}{$6-311+\mathrm{G}(3 \mathrm{df})$} & $\mathrm{uC}-6-311+\mathrm{G}(3 \mathrm{df})$ & $6-311 \mathrm{G}$ & -191.608320 & 539.5005 \\
\hline & $\mathrm{u}-6-311+\mathrm{G}(3 \mathrm{df})$ & & -191.611347 & 539.3078 \\
\hline aug-cc-pVQZ & & aug-cc-pVTr & -191.712598 & 539.2886 \\
\hline aug-cc-pVTZ & aug-cc-pV5Z & aug-cc-pVTr & -191.661197 & 539.2722 \\
\hline aug-cc-pVTZ & & aug-cc-pVD' & -191.643593 & 539.2678 \\
\hline aug-cc-pVQZ & & aug-cc-pVTr & -191.712657 & 539.2502 \\
\hline aug-cc-pVTZ & uC-aug-cc-pV5Z & aug-cc-pVTr & -191.661307 & 539.2325 \\
\hline aug-cc-pVTZ & & aug-cc-pVD' & -191.643713 & 539.2278 \\
\hline
\end{tabular}

to $0.03-0.04 \mathrm{eV}$ drop in IE. Let us first discuss the results for the oxygen edge. In these calculations, our largest basis for the inactive edge was aug-cc-pVQZ. Further reducing this basis to aug-cc-pVTZ leads to a change of $0.02 \mathrm{eV}$. The effect of the basis on the hydrogen is even smaller - for example, reducing the basis on hydrogens from triple- $\zeta$ to double- $\zeta$ changes the IEs by $0.005 \mathrm{eV}$ only. The trend in IEs computed with Pople's bases is similar to the previous cases. The results for $\mathrm{u}-6-311+\mathrm{G}(3 \mathrm{df}) / 6-311+\mathrm{g}(3 \mathrm{df}) / 6-311 \mathrm{G}$ are within $0.06 \mathrm{eV}$ from the uC-aug-cc-pV5Z/aug-cc-pVQZ/aug-cc-pVTZ (our largest basis in this calculation). The total number of basis functions in these two calculations are 181 and 467, respectively.

The results for the carbon edge, shown in Table XI, follow similar trends. We observe that the chemical shifts (the difference between $1 s_{C}$ IEs relative to $\mathrm{C} 1$ ) converge with respect to the basis much faster than the absolute values. As said, the largest basis used in this calculation is uC-aug-cc-pV5Z/aug-cc-pVTZ/aug-cc-pVTZ. The results for $\mathrm{u}-6-311+\mathrm{G}(3 \mathrm{df}) / 6$ $311+\mathrm{g}(3 \mathrm{df}) / 6-311 \mathrm{G}$ are within $0.02 \mathrm{eV}$ from that value. Comparing our best estimates to the experiment (only the shifts were reported in Ref. [54]), we note excellent agreement for C3 (2.63 eV versus $2.6 \mathrm{eV})$, however, for the $\mathrm{C} 2$ shift we consistently obtain $\sim 0.3 \mathrm{eV}$, versus zero shift reported in Ref. [54].

\section{G. Glycine}

Glycine $\left(\mathrm{C}_{2} \mathrm{H}_{5} \mathrm{NO}_{2}\right.$, canonical form shown in Fig. 2) is a polyatomic molecule featuring multiple core IEs and three different edges[25, 55, 56]. Table XII shows the results for the mixed basis sets in which we used uC-aug-cc-pV5Z for the active edge and aug-cc-pVTZ 
TABLE XI: Acrolein, core IEs for the carbon edge.

\begin{tabular}{lllll} 
Basis on C & Basis on O & Basis on H & CCSD energy $^{a}(\mathrm{a.u})$ & $\mathrm{IE}(\mathrm{eV})^{b}$ Shift $(\mathrm{eV})^{c}$ \\
\hline 6-311+G(3df) & 6-311+G(3df) $6-311 \mathrm{G}$ & -191.538887 & 291.90130 .0 \\
& & & 292.19800 .2967 \\
& & & 294.54332 .6420 \\
$\mathrm{uC}-6-311+\mathrm{G}(3 \mathrm{df})$ & $6-311+\mathrm{G}(3 \mathrm{df})$ 6-311G & -191.541545 & 291.63300 .0 \\
& & & 291.92160 .2886 \\
$\mathrm{u}-6-311+\mathrm{G}(3 \mathrm{df})$ & $6-311+\mathrm{G}(3 \mathrm{df})$ 6-311G & -191.545560 & 294.25882 .6258 \\
& & & 291.50790 .0 \\
& & & 291.78720 .2793 \\
& & & 294.14882 .6409 \\
\hline
\end{tabular}

aug-cc-pVQZ aug-cc-pVTZ aug-cc-pVTZ -191.601682 291.63210 .00

291.90260 .2705

294.26492 .6328

uC-aug-cc-pVQZ aug-cc-pVTZ aug-cc-pVTZ -191.602010

291.54530 .00

291.81550 .2702

294.17302 .6277

aug-cc-pV5Z aug-cc-pVTZ aug-cc-pVTZ -191.611970

291.53640 .00

291.80710 .2707

294.16502 .6286

aug-cc-pV5Z aug-cc-pVTZ aug-cc-pVDZ -191.606131

291.52980 .00

291.79910 .2693

294.15852 .6287

uC-aug-cc-pV5Z aug-cc-pVTZ aug-cc-pVTZ -191.612021

291.50860 .00

291.77940 .2708

294.13592 .6273

uC-aug-cc-pV5Z aug-cc-pVTZ aug-cc-pVDZ -191.606239

291.50120 .00

291.77020 .2690

294.12862 .6274

$\bar{a}$ Total energy for the neutral reference state. ${ }^{b}$ The IEs are arranged in the order C2, C1, and C3, refer to Fig. 1. ${ }^{c}$ Experimental shifts in IEs are 0.0, 0.0, and 2.6 with respect to C1.

basis for other heavy atoms. For the hydrogens, we used aug-cc-pVDZ and aug-cc-pVTZ. Similarly to the acrolein example, the difference in IEs between these calculations is 0.01 $\mathrm{eV}$. 
Table XIII shows the results with Pople's bases. As in other cases, we see that the u-6$311+\mathrm{G}(3 \mathrm{df}) / 6-311+\mathrm{G}(3 \mathrm{df}) / 6-311 \mathrm{G}$ results are within $0.07 \mathrm{eV}$ from our best estimates. We also performed calculations with the fully uncontracted Pople bases on all atoms and, as in previous cases did not observe much difference (results not shown). Finally, Fig. 6 compares the selected results against the available experimental values and our best estimate. The IEs computed with $\mathrm{u}-6-311+\mathrm{G}(3 \mathrm{df}) / 6-311+\mathrm{G}(3 \mathrm{df}) / 6-311 \mathrm{G}$ are within $0.2 \mathrm{eV}$ for oxygen and carbon edges, and $1 \mathrm{eV}$ for nitrogen edge from the experiments. The shifts between $\mathrm{C} 1 / \mathrm{C} 2$ and $\mathrm{O} 1 / \mathrm{O} 2$ are also reproduced well.

TABLE XII: Glycine core IEs for all edges with mixed basis sets ${ }^{a}$.

\begin{tabular}{|c|c|c|}
\hline \multicolumn{3}{|l|}{ C Edge } \\
\hline Basis on H $\quad$ CCSD energy $^{b}$ (a.u.) & IE C1 (eV) & IE C2 (eV) \\
\hline aug-cc-pVDZ -284.013614 & 292.5430 & 295.1946 \\
\hline aug-cc-pVTZ -284.027506 & 292.5531 & 295.1988 \\
\hline \multicolumn{3}{|l|}{ O Edge } \\
\hline CCSD energy $^{b}$ (a.u.) & IE O1 (eV) & $\mathrm{IE} \mathrm{O} 2(\mathrm{eV})$ \\
\hline aug-cc-pVDZ -284.089201 & 538.6577 & 540.2119 \\
\hline aug-cc-pVTZ -284.110647 & 538.6632 & 540.2216 \\
\hline \multicolumn{3}{|l|}{ N Edge } \\
\hline CCSD Energy $^{b}$ (a.u.) & \multicolumn{2}{|c|}{ IE N (eV) } \\
\hline aug-cc-pVDZ -284.037027 & \multicolumn{2}{|c|}{406.5789} \\
\hline aug-cc-pVTZ -284.053208 & \multicolumn{2}{|c|}{406.5922} \\
\hline
\end{tabular}

${ }^{a}$ Active edge basis: $\mathrm{uC}$-aug-cc-pV5Z, inactive edge basis: aug-cc-pVTZ. ${ }^{b}$ Total energy for the neutral reference state. 
TABLE XIII: Glycine core IEs with Pople basis sets.

\begin{tabular}{|c|c|c|c|}
\hline \multicolumn{4}{|c|}{ C Edge ${ }^{a}$} \\
\hline Basis on active edq & CCSD Energy $^{b}$ (a.u.) & IE C1 (eV) & $\mathrm{IE} \mathrm{C} 2(\mathrm{eV})$ \\
\hline $6-311+\mathrm{G}(2 \mathrm{df})$ & -283.922367 & 293.0310 & 295.6453 \\
\hline $\mathrm{uC}-6-311+\mathrm{G}(2 \mathrm{df})$ & -283.924455 & 292.7187 & 295.3349 \\
\hline $\mathrm{u}-6-311+\mathrm{G}(2 \mathrm{df})$ & -283.927506 & 292.5870 & 295.2341 \\
\hline $6-311+\mathrm{G}(3 \mathrm{df})$ & -283.941538 & 293.0077 & 295.5965 \\
\hline $\mathrm{uC}-6-311+\mathrm{G}(3 \mathrm{df})$ & -283.943366 & 292.7032 & 295.3202 \\
\hline $\mathrm{u}-6-311+\mathrm{G}(3 \mathrm{df})$ & -283.946117 & 292.5704 & 295.2269 \\
\hline \multicolumn{4}{|c|}{$\mathrm{O} \mathrm{Edge}^{a}$} \\
\hline Basis & CCSD Energy (a.u.) & \multicolumn{2}{|c|}{ IE O1 (eV) IE O2 (eV) } \\
\hline $6-311+\mathrm{G}(2 \mathrm{df})$ & -283.986182 & 539.5400 & 541.0882 \\
\hline $\mathrm{uC}-6-311+\mathrm{G}(2 \mathrm{df})$ & -283.991309 & 538.9286 & 540.4764 \\
\hline $\mathrm{u}-6-311+\mathrm{G}(2 \mathrm{df})$ & -283.997698 & 538.7314 & 540.2612 \\
\hline $6-311+\mathrm{G}(3 \mathrm{df})$ & -284.009612 & 539.4839 & 541.0594 \\
\hline $\mathrm{uC}-6-311+\mathrm{G}(3 \mathrm{df})$ & -284.014362 & 538.9247 & 540.4761 \\
\hline $\mathrm{u}-6-311+\mathrm{G}(3 \mathrm{df})$ & -284.020468 & 538.7336 & 540.2695 \\
\hline \multicolumn{4}{|c|}{$\mathrm{N} \mathrm{Edge}^{a}$} \\
\hline Basis & CCSD Energy (a.u.) & \multicolumn{2}{|c|}{ IE N (eV) } \\
\hline $6-311+\mathrm{G}(2 \mathrm{df})$ & -283.964956 & \multicolumn{2}{|c|}{407.3069} \\
\hline $\mathrm{uC}-6-311+\mathrm{G}(2 \mathrm{df})$ & -283.966768 & \multicolumn{2}{|c|}{406.8375} \\
\hline $\mathrm{u}-6-311+\mathrm{G}(2 \mathrm{df})$ & -283.969069 & \multicolumn{2}{|c|}{406.6510} \\
\hline $6-311+\mathrm{G}(3 \mathrm{df})$ & -283.986780 & \multicolumn{2}{|c|}{407.2477} \\
\hline $\mathrm{uC}-6-311+\mathrm{G}(3 \mathrm{df})$ & -283.988335 & \multicolumn{2}{|c|}{406.8096} \\
\hline $\mathrm{u}-6-311+\mathrm{G}(3 \mathrm{df})$ & -283.990529 & \multicolumn{2}{|c|}{406.6262} \\
\hline
\end{tabular}

${ }^{a}$ See Fig. 2 for notations. ${ }^{b}$ Total energy for the neutral reference state. ${ }^{c}$ Inactive edge and $\mathrm{H}$ basis is the contracted version of the basis on active edge Experimental IEs: C1:

$292.3 \mathrm{eV}, \mathrm{C} 2: 295.2 \mathrm{eV}, \mathrm{O} 1: 538.4 \mathrm{eV}, \mathrm{O} 2: 540.2 \mathrm{eV}, \mathrm{N}: 405.4 \mathrm{eV}$ (from Ref. [56]).

\section{CONCLUSION}

We presented a computational study of basis-set effects in calculations of core-ionized states using a correlated method, fc-CVS-EOM-IP-CCSD. In agreement with previous studies, we observed that core-level states require higher-quality basis sets than valence states because of the large perturbation on the electronic structure due to removal of a core electron. Although the converged results can be obtained by using very large Dunning's bases, such as aug-cc-pCV5Z, we investigated a different strategy, that is, using core- and fully uncontracted basis sets. Our results indicate that this approach is much more effective. We 


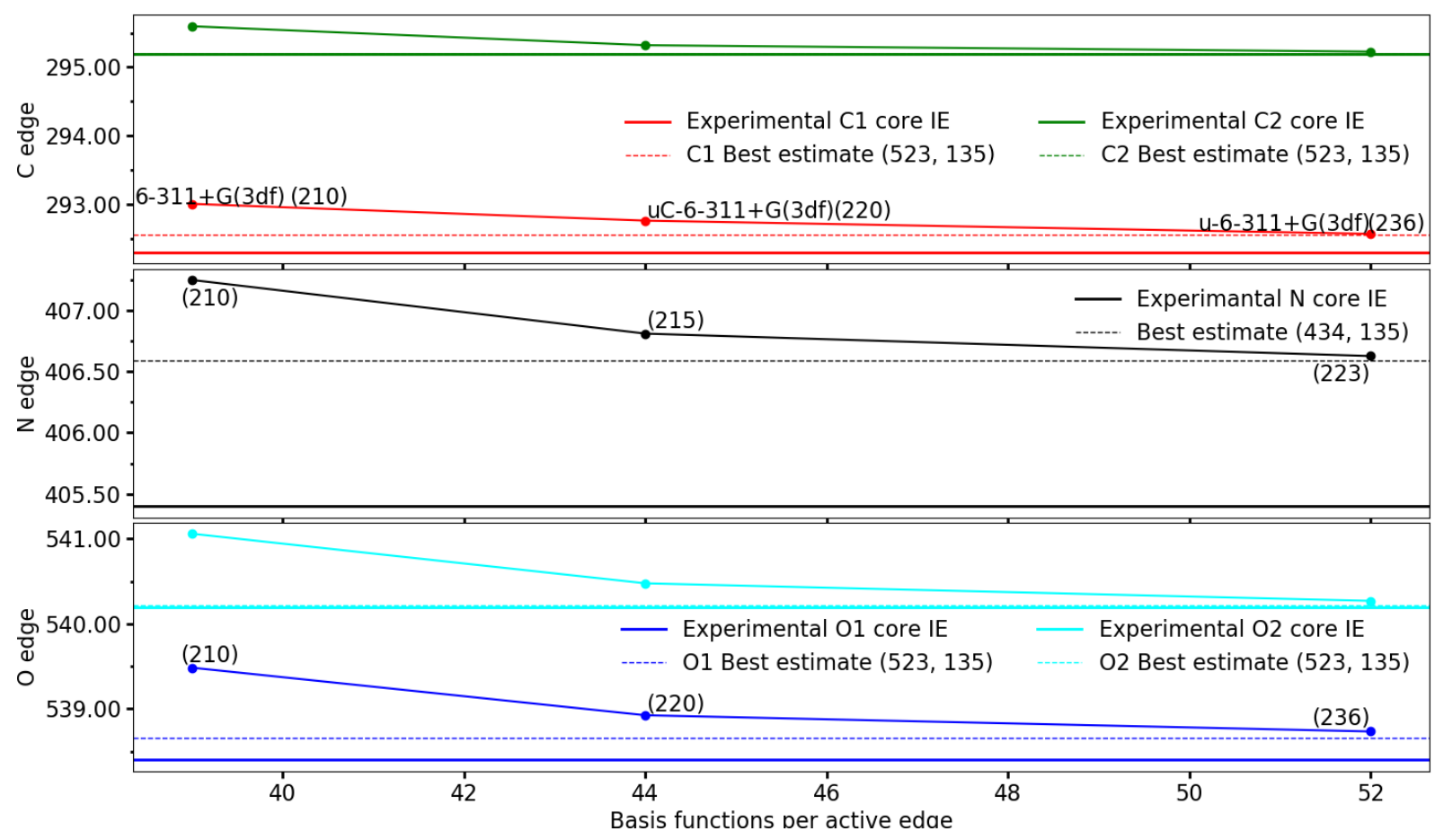

FIG. 6: Glycine IEs for the carbon, nitrogen and oxygen edges versus the number of basis functions per active edge atom. The total number of basis functions in each calculation is shown in parentheses in the respective panel. The best estimate is obtained with the uC-aug-cc-pV5Z/augcc-pVTZ/aug-cc-pVTZ basis; the respective total number of basis functions and the number of basis functions per active edge atom are shown in parentheses.

observe especially good performance for uncontracted Pople's bases. For example, the results with $\mathrm{u}-6-311 \mathrm{G}+(3 \mathrm{df})$ are of nearly the same quality as with aug-cc-pV5Z, despite having $60 \%$ less basis functions. For the systems we studied, the results with uC-6-311+G(3df) and $\mathrm{u}-6-311+\mathrm{G}(3 \mathrm{df})$ are within $0.07 \mathrm{eV}$ from the basis-set limit. These errors are smaller than the anticipated errors due to an incomplete treatment of electron correlation. Slightly smaller bases, $\mathrm{uC}-6-311+\mathrm{G}(2 \mathrm{df})$ and $\mathrm{u}-6-311+\mathrm{G}(2 \mathrm{df})$ also perform very well. Thus, our recommended approach to core-level calculations is to use the uncontracted variants of the standard bases. The largest gain is achieved by uncontracting the core. The results show that it is sufficient to uncontract only the basis used for the active edge, while treating the rest of the atoms with matching contracted bases. Smaller bases can be used on hydrogens, without significant effect on the core IEs.

We also investigated more aggressive cost-saving strategies: using mixed bases on active and inactive edges, using single-precision at the CCSD step, and using the FNO-based truncation of the virtual space. The results pave a way towards cost-effective and accurate 
calculations of core-level states.

\section{Acknowledgments}

We are grateful to Prof. Peter Gill from the University of Sydney for his insightful remarks on the physics of core-ionized states and anticipated consequences for basis set selection, which motivated the present study.

This work was supported by the U.S. National Science Foundation (No. CHE-1856342). A.I.K. is a grateful recipient of the Simons Fellowship in Theoretical Physics and Mildred Dresselhaus Award from CFEL/DESY, which supported her sabbatical stay in Germany. M.L.V. and S.C. acknowledge financial support from DTU Chemistry and from the Independent Research Fund Denmark-Natural Sciences, DFF-RP2 grant no. 7014-00258B. S.C. also acknowledges the European Union's Horizon 2020 Research and Innovation Programme under the Marie Sklodowska-Curie Grant Agreement No. 765739, "COSINE- European Training Network on COmputational Spectroscopy In Natural sciences and Engineering."

\section{Conflicts of interest}

A.I.K. is the President and a part-owner of Q-Chem, Inc. 
[1] S. Mobilio, F. Boscherini, and C. Meneghini, editors, Synchrotron Radiation: Basics, Methods and Applications. Springer, 2014.

[2] J.A. van Bokhoven and C. Lamberti, editors, X-Ray Absorption and X-ray Emission Spectroscopy; Theory and Applications. Wiley \& Sons, 2016.

[3] U. Bergmann, V. K. Yachandra, and J. Yano, editors, X-Ray Free Electron Lasers: Applications in Materials, Chemistry and Biology, Number 18 in Energy and Environment Series. Royal Society of Chemistry, 2017.

[4] M. Nisoli, P. Decleva, F. Calegari, A. Palacios, and F. Martín, Attosecond electron dynamics in molecules, Chem. Rev. 117, 10760 (2017).

[5] M. Ahmed and O. Kostko, From atoms to aerosols: probing clusters and nanoparticles with synchrotron based mass spectrometry and X-ray spectroscopy, Phys. Chem. Chem. Phys. 22, $2713(2020)$.

[6] O. Kostko, B. Bandyopadhyay, and M. Ahmed, Vacuum ultraviolet photoionization of complex chemical systems, Annu. Rev. Phys. Chem. 67, 19 (2016).

[7] P. Norman and A. Dreuw, Simulating X-ray spectroscopies and calculating core-excited states of molecules, Chem. Rev. 118, 7208 (2018).

[8] T. Fransson, Y. Harada, N. Kosugi, N. A. Besley, B. Winter, J. J. Rehr, L. G. M. Pettersson, and A. Nilsson, X-ray and electron spectroscopy of water, Chem. Rev. 116, 7551 (2016).

[9] J. A. Pople, Theoretical models for chemistry, in Energy, Structure and Reactivity: Proceedings of the 1972 Boulder Summer Research Conference on Theoretical Chemistry, edited by D.W. Smith and W.B. McRae, pages 51-61. Wiley, New York, 1973.

[10] K. Emrich, An extension of the coupled-cluster formalism to excited states (I), Nucl. Phys. A351, 379 (1981).

[11] J. F. Stanton and R. J. Bartlett, The equation of motion coupled-cluster method. A systematic biorthogonal approach to molecular excitation energies, transition probabilities, and excited state properties, J. Chem. Phys. 98, 7029 (1993).

[12] A. I. Krylov, Equation-of-motion coupled-cluster methods for open-shell and electronically excited species: The hitchhiker's guide to Fock space, Annu. Rev. Phys. Chem. 59, 433 (2008).

[13] R. J. Bartlett, The coupled-cluster revolution, Mol. Phys. 108, 2905 (2010). 
[14] K. Sneskov and O. Christiansen, Excited state coupled cluster methods, WIREs: Comput. Mol. Sci. 2, 566 (2012).

[15] R. J. Bartlett, Coupled-cluster theory and its equation-of-motion extensions, WIREs: Comput. Mol. Sci. 2, 126 (2012).

[16] N.A. Besley, A.T.B. Gilbert, and P.M.W. Gill, Self-consistent-field calculations of core excited states, J. Chem. Phys. 130, 124308 (2009).

[17] M. Nooijen and R. J. Bartlett, Description of core-excitation spectra by the open-shell electron-attachment equation-of-motion coupled cluster method, J. Chem. Phys. 102, 6735 (1995).

[18] S. Coriani, O. Christiansen, T. Fransson, and P. Norman, Coupled-cluster response theory for near-edge x-ray-absorption fine structure of atoms and molecules, Phys. Rev. A 85, 022507 (2012).

[19] S. Coriani, T. Fransson, O. Christiansen, and P. Norman, Asymmetric-Lanczos-chain-driven implementation of electronic resonance convergent coupled-cluster linear response theory, J. Chem. Theory Comput. 8, 1616 (2012).

[20] T. J. Watson and R. J. Bartlett, Infinite order relaxation effects for core ionization energies with a variational coupled cluster ansatz, Chem. Phys. Lett. 555, 235 (2013).

[21] S. Sen, A. Shee, and D. Mukherjee, A study of the ionisation and excitation energies of core electrons using a unitary group adapted state universal approach, Mol. Phys. 11, 2625 (2013).

[22] D. Zuev, E. Vecharynski, C. Yang, N. Orms, and A. I. Krylov, New algorithms for iterative matrix-free eigensolvers in quantum chemistry, J. Comput. Chem. 36, 273 (2015).

[23] B. Peng, P.J. Lestrange, J.J. Goings, M. Caricato, and X. Li, Energy-specific equation-ofmotion coupled-cluster methods for high-energy excited states: Application to K-edge X-ray absorption spectroscopy, J. Chem. Theory Comput. 11, 4146 (2015).

[24] S. Coriani and H. Koch, Communication: X-ray absorption spectra and core-ionization potentials within a core-valence separated coupled cluster framework, J. Chem. Phys. 143, 181103 (2015).

[25] A. Sadybekov and A. I. Krylov, Coupled-cluster based approach for core-ionized and coreexcited states in condensed phase: Theory and application to different protonated forms of aqueous glycine, J. Chem. Phys. 147, 014107 (2017).

[26] R. H. Myhre, S. Coriani, and H. Koch, Near-edge X-ray absorption fine structure within 
multilevel coupled cluster theory, J. Chem. Theory Comput. 12, 2633 (2016).

[27] M. L. Vidal, X. Feng, E. Epifanovski, A. I. Krylov, and S. Coriani, A new and efficient equation-of-motion coupled-cluster framework for core-excited and core-ionized states, J. Chem. Theory Comput. 15, 3117 (2019).

[28] B. N. C. Tenorio, T. Moitra, M. A. C. Nascimento, A. B. Rocha, and S. Coriani, Molecular inner-shell photoabsorption/photoionization cross sections at core-valence-separated coupled cluster level: Theory and examples, J. Chem. Phys. 150, 224104 (2019).

[29] K. Nanda, M. L. Vidal, R. Faber, S. Coriani, and A. I. Krylov, How to stay out of trouble in RIXS calculations within the equation-of-motion coupled-cluster damped response theory framework? Safe hitchhiking in the excitation manifold by means of core-valence separation, Phys. Chem. Chem. Phys. 22, 2629 (2020).

[30] R. Faber and S. Coriani, Resonant inelastic X-ray scattering and nonesonant X-ray emission spectra from coupled-cluster (damped) response theory, J. Chem. Theory Comput. 15, 520 (2019).

[31] R. Faber and S. Coriani, Core-valence-separated coupled-cluster-singles-and-doubles complexpolarization-propagator approach to X-ray spectroscopies, Phys. Chem. Chem. Phys. 22, 2642 (2020).

[32] L. S. Cederbaum, W. Domcke, and J. Schirmer, Many-body theory of core holes, Phys. Rev. A 22, 206 (1980).

[33] S. Coriani and H. Koch, Erratum: "Communication: X-ray absorption spectra and coreionization potentials within a core-valence separated coupled cluster framework" [J. Chem. Phys. 143, 181103 (2015)], J. Chem. Phys. 145, 149901 (2016).

[34] F. Frati, F. de Groot, J. Cerezo, F. Santoro, L. Cheng, R. Faber, and S. Coriani, Coupled cluster study of the x-ray absorption spectra of formaldehyde derivatives at the oxygen, carbon, and fluorine K-edges, J. Chem. Phys. 151, 064107 (2019).

[35] J. P. Carbonea, L. Cheng, R. H. Myhre, D. Matthews, H. Koch, and S. Coriani, Advances in Quantum Chemistry, volume 79, chapter An analysis of the performance of coupled cluster methods for K-edge core excitations and ionizations using standard basis sets, pages 241-261. Elsevier Inc, 2019.

[36] J. Wenzel, M. Wormit, and A. Dreuw, Calculating core-level excitations and x-ray absorption spectra of medium-sized closed-shell molecules with the algebraic-diagrammatic construction 
scheme for the polarization propagator, J. Comput. Chem. 35, 1900 (2014).

[37] S. Shirai, S. Yamamoto, and S. Hyodo, Accurate calculation of core-electron binding energies: Multireference perturbation treatment, J. Chem. Phys. 121, 7586 (2004).

[38] Y. Takahata and D. P. Chong, DFT calculation of core-electron binding energies, J. Electron. Spectrosc. Relat. Phenom. 133, 69 (2003).

[39] J. Wenzel, A. Holzer, M. Wormit, and A. Dreuw, Analysis and comparison of CVS-ADC approaches up to third order for the calculation of core-excited states, J. Chem. Phys. 142, $214104(2015)$.

[40] J. Liu, D. Matthews, S. Coriani, and L. Cheng, Benchmark calculations of K-edge ionization energies for first-row elements using scalar-relativistic corevalence-separated equation-ofmotion coupled-cluster methods, J. Chem. Theory Comput. 15, 1642 (2019).

[41] M. L. Vidal, A. I. Krylov, and S. Coriani, Dyson orbitals within the fc-CVS-EOM-CCSD framework: theory and application to X-ray photoelectron spectroscopy of ground and excited states, Phys. Chem. Chem. Phys. 22, 2693 (2020).

[42] M. L. Vidal, A. I. Krylov, and S. Coriani, Correction to: "Dyson orbitals within the fcCVS-EOM-CCSD framework: theory and application to X-ray photoelectron spectroscopy of ground and excited states", Phys. Chem. Chem. Phys. 22, 3744 (2020).

[43] J. C. Slater, Atomic schielding constants, Phys. Rev. 36, 57 (1930).

[44] L. Kjellsson, K. Nanda, J.-E. Rubensson, G. Doumy, S. H. Southworth, P. J. Ho, A. M. March, A. Al Haddad, Y. Kumagai, M.-F. Tu, T. Debnath, M. S. Bin Mohd Yusof, C. Arnold, W. F. Schlotter, S. Moeller, G. Coslovich, J. D. Koralek, M. P. Minitti, M. L. Vidal, M. Simon, R. Santra, Z.-H. Loh, S. Coriani, A. I. Krylov, and L. Young, RIXS reveals hidden local transitions of the aqueous OH radical, Phys. Rev. Lett. (2020), submitted; https://arxiv.org/abs/2003.03909.

[45] Y. Shao, Z. Gan, E. Epifanovsky, A.T.B. Gilbert, M. Wormit, J. Kussmann, A.W. Lange, A. Behn, J. Deng, X. Feng, D. Ghosh, M. Goldey, P.R. Horn, L.D. Jacobson, I. Kaliman, R.Z. Khaliullin, T. Kus, A. Landau, J. Liu, E.I. Proynov, Y.M. Rhee, R.M. Richard, M.A. Rohrdanz, R.P. Steele, E.J. Sundstrom, H.L. Woodcock III, P.M. Zimmerman, D. Zuev, B. Albrecht, E. Alguires, B. Austin, G.J.O. Beran, Y.A. Bernard, E. Berquist, K. Brandhorst, K.B. Bravaya, S.T. Brown, D. Casanova, C.-M. Chang, Y. Chen, S.H. Chien, K.D. Closser, D.L. Crittenden, M. Diedenhofen, R.A. DiStasio Jr., H. Do, A.D. Dutoi, R.G. Edgar, S. 
Fatehi, L. Fusti-Molnar, A. Ghysels, A. Golubeva-Zadorozhnaya, J. Gomes, M.W.D. HansonHeine, P.H.P. Harbach, A.W. Hauser, E.G. Hohenstein, Z.C. Holden, T.-C. Jagau, H. Ji, B. Kaduk, K. Khistyaev, J. Kim, J. Kim, R.A. King, P. Klunzinger, D. Kosenkov, T. Kowalczyk, C.M. Krauter, K.U. Laog, A. Laurent, K.V. Lawler, S.V. Levchenko, C.Y. Lin, F. Liu, E. Livshits, R.C. Lochan, A. Luenser, P. Manohar, S.F. Manzer, S.-P. Mao, N. Mardirossian, A.V. Marenich, S.A. Maurer, N.J. Mayhall, C.M. Oana, R. Olivares-Amaya, D.P. O’Neill, J.A. Parkhill, T.M. Perrine, R. Peverati, P.A. Pieniazek, A. Prociuk, D.R. Rehn, E. Rosta, N.J. Russ, N. Sergueev, S.M. Sharada, S. Sharmaa, D.W. Small, A. Sodt, T. Stein, D. Stuck, Y.-C. Su, A.J.W. Thom, T. Tsuchimochi, L. Vogt, O. Vydrov, T. Wang, M.A. Watson, J. Wenzel, A. White, C.F. Williams, V. Vanovschi, S. Yeganeh, S.R. Yost, Z.-Q. You, I.Y. Zhang, X. Zhang, Y. Zhou, B.R. Brooks, G.K.L. Chan, D.M. Chipman, C.J. Cramer, W.A. Goddard III, M.S. Gordon, W.J. Hehre, A. Klamt, H.F. Schaefer III, M.W. Schmidt, C.D. Sherrill, D.G. Truhlar, A. Warshel, X. Xu, A. Aspuru-Guzik, R. Baer, A.T. Bell, N.A. Besley, J.-D. Chai, A. Dreuw, B.D. Dunietz, T.R. Furlani, S.R. Gwaltney, C.-P. Hsu, Y. Jung, J. Kong, D.S. Lambrecht, W.Z. Liang, C. Ochsenfeld, V.A. Rassolov, L.V. Slipchenko, J.E. Subotnik, T. Van Voorhis, J.M. Herbert, A.I. Krylov, P.M.W. Gill, and M. Head-Gordon, Advances in molecular quantum chemistry contained in the Q-Chem 4 program package, Mol. Phys. 113, $184(2015)$.

[46] A. I. Krylov and P. M. W. Gill, Q-Chem: An engine for innovation, WIREs: Comput. Mol. Sci. 3, 317 (2013).

[47] P. U. Manohar, J. F. Stanton, and A. I. Krylov, Perturbative triples correction for the equation-of-motion coupled-cluster wave functions with single and double substitutions for ionized states: Theory, implementation, and examples, J. Chem. Phys. 131, 114112 (2009).

[48] B. P. Pritchard, D. Altarawy, B. Didier, T. D. Gibson, and T. L. Windus, New basis set exchange: An open, up-to-date resource for the molecular sciences community, J. Chem. Inf. Model. 59, 4814 (2019).

[49] W.L. Jolly, K.D. Bomben, and C.J. Eyermann, Core-electron binding energies for gaseous atoms and molecules, Atomic Data and Nuclear Data Tables 31, 433 (1984).

[50] J. Schirmer, A. Trofimov, K. Randall, J. Feldhaus, A. M. Bradshaw, Y. Ma, C.T. Chen, and F. Sette, K-shell excitation of the water, ammonia, and methane molecules using highresolution photoabsorption spectroscopy, Phys. Rev. A 47, 1136 (1993). 
[51] P. Pokhilko, E. Epifanovskii, and A. I. Krylov, Double precision is not needed for many-body calculations: Emergent conventional wisdom, J. Chem. Theory Comput. 14, 4088 (2018).

[52] A. Landau, K. Khistyaev, S. Dolgikh, and A. I. Krylov, Frozen natural orbitals for ionized states within equation-of-motion coupled-cluster formalism, J. Chem. Phys. 132, 014109 (2010).

[53] P. Pokhilko, D. Izmodenov, and A. I. Krylov, Extension of frozen natural orbital approximation to open-shell references: Theory, implementation, and application to single-molecule magnets, J. Chem. Phys. 152, 034105 (2020).

[54] D. Duflot, J.-P. Flament, I. C. Walker, J. Heinesch, and M.-J. Hubin-Franskin, Core shell excitation of 2-propenal (acrolein) at the $\mathrm{O}$ 1s and $\mathrm{C}$ 1s edges: An experimental and ab initio study, J. Chem. Phys. 118, 1137 (2003).

[55] R. H. Myhre, S. Coriani, and H. Koch, X-ray and UV spectra of glycine within coupled cluster linear response theory, J. Phys. Chem. A 123, 9701 (2019).

[56] O. Plekan, V. Feyer, R. Richter, M. Coreno, M. de Simone, K. C. Prince, and V. Carravetta, Investigation of the amino acids glycine, proline, and methionine by photoemission spectroscopy, J. Phys. Chem. A 111, 10998 (2007). 